\title{
Ant colony optimization for slope stability analysis applied to an embankment failure in eastern India
}

\author{
Mayank Mishra ${ }^{1 *}$, Mandeep Singh Basson², Gunturi Venkata Ramana ${ }^{3}$ and Roberto Vassallo ${ }^{4}$
}

*Correspondence:
mayank@iitbbs.ac.in
' School of Infrastructure,
Indian Institute
of Technology Bhubaneswar,
Argul, Khordha 752050, India
Full list of author information
is available at the end of the
article

article

\begin{abstract}
The safety of road embankments is mainly assessed in the engineering practice by limit equilibrium analyses. Locating the critical slip surface of embankments and calculating the corresponding factor of safety is a crucial task. In this paper, the continuous ant colony optimisation algorithm is used to analyse the stability of slopes with noncircular slip surfaces. To illustrate the proposed procedure, one example from published literature and another engineering case study of a landslide at a road construction site in India is analysed. This latter study is supplemented by the results of geotechnical investigations performed before and after the failure of the embankment. The results demonstrate that the proposed technique identifies correctly the critical slip surface and can thus be used for engineering applications.
\end{abstract}

Keywords: Slope stability, Landslide, Continuous ant colony optimisation, Critical slip surface, Case study

\section{Introduction}

Slope stability assessment is regularly carried out by engineers to analyse the stability of natural or man-made slopes and understand their failure mechanisms. In slope stability assessment, it is essential to determine the critical slip surface, i.e. the one characterised by the lowest safety factor, and, in professional practice, the analysis is routinely carried out using Limit Equilibrium Methods (LEM). Unstable conditions can be induced by several causes such as excessive surcharge load, earthquakes, heavy rainfall and dynamic loads. They can lead to slope failures causing considerable damage to structures and infrastructure facilities arising on them. The task is to find the minimum factor of safety against all these destabilising factors.

The most widely used methods in slope stability assessment are the traditional limit equilibrium methods [1-4]. In addition, finite difference technique [5, 6], distinct element codes [7] and finite element strength reduction technique (FEM) [8-13] are also used extensively. Use of optimisation methods such as dynamic programming [14], downhill simplex algorithm [15] and Monte-Carlo (MC) technique [16] are also reported. Recently, other optimisation techniques inspired by nature like ant colony optimisation (ACO) [17-19], particle swarm optimisation (PSO) [20, 21], harmony

(c) The Author(s) 2020. This article is licensed under a Creative Commons Attribution 4.0 International License, which permits use, sharing adaptation, distribution and reproduction in any medium or format, as long as you give appropriate credit to the original author(s) and the source, provide a link to the Creative Commons licence, and indicate if changes were made. The images or other third party material in this article are included in the article's Creative Commons licence, unless indicated otherwise in a credit line to the material. If material is not included in the article's Creative Commons licence and your intended use is not permitted by statutory regulation or exceeds the permitted use, you will need to obtain permission directly from the copyright holder. To view a copy of this licence, visit http://creativeco mmons.org/licenses/by/4.0/. 
search (HS) [22], artificial bee colony algorithm (ABC) [23], gravitational search algorithm (GSA) [24], neural networks (NN) [25, 26] and genetic algorithms (GA) [27-29] have received considerable attention and are gaining popularity in the geotechnical engineering community in the last $10-15$ years.

Cheng et al. [30] compared six metaheuristic optimisation methods and reported that no single method can outperform other methods in all aspects, each one having its own merits and limitations. In all these methods, it is paramount first to identify the critical slip surface. This critical slip surface is found by comparing the factors of safety obtained from several feasible trial surfaces and choosing the one with the minimum value. This may sometimes be erroneous due to the presence of local minima points. Many existing methods fail to identify the global minimum and may converge at a local minima even for simple homogenous slopes [31]. Neural networks on the other hand are site specific, require reliable training data and apply for particular cases only [25, 32, 33]. Discrete ACO used previously for FS calculation takes too many fitness function evaluations to compute optimum FS and has more uncertainty in FS estimation. Therefore several global optimisation algorithms are available to locate critical slip surfaces but are either too complex to implement for practitioners based on an excessive number of fitness function evaluations. The use of finite element techniques bypasses the need to assume the shape and location of the trial slip surfaces, but also introduces other complexities including defining appropriate soil constitutive models.

Most of the existing software packages available for slope stability calculations [34-36] implement a method of slip surface search called point grid approach. The location of the centre point of the slip surface, that in this case is assumed as circular, is shifted during the search until it corresponds to the lowest factor of safety. However, this method does not assure that the slip surface retrieved corresponds the global minimum. Some programs [37] implements cuckoo search [38] and simulated annealing [39] for noncircular failure surfaces. Monte-Carlo search techniques are also implemented in limit equilibrium slope stability programs (SAS-MCT 4.0) [40]. For evolutionary computation, codes such as Slope SGA [29, 41] incorporate a Genetic Algorithm (GA) for both circular and non-circular slip surfaces. The commercially available software Geostudio [35] uses an optimising function which is based on search techniques used by Greco [16] and Malkawi et al. [40] to search for critical slip surface. Apart from that, to the authors' knowledge, there is no other commercial program using intelligent algorithms optimisation techniques for slope stability analysis. Published research $[17,18,30]$ demonstrates that there is a growing interest in ant-based algorithm metaheuristic for slope stability assessment.

Most of the intelligent algorithms for slope stability calculations are relatively new, difficult to implement and not familiar to geotechnical engineers. Sengupta et al. [27] used a circular surface with the genetic algorithm to find the location of the critical slip surface and the corresponding factor of safety using Bishop's method of slices [1]. The factor of safety was defined as a fitness function expressed in terms of coordinates of the centre of the slip surface and its radius (three control variables). For a non-circular slip surface, the control variables will be more than three. Kahatadeniya et al. [17] used discrete ant colony optimisation and Kashani et al. used [42] Imperialistic Competitive Algorithm (ICA) to determine the optimal curve yielding 
minimum factor of safety using Morgenstern-Price method [3]. The ant colony optimisation technique used by Kahatadeniya et al. [17] was in discrete search space and required careful calibration of parameters to arrive to correct results. Furthermore by using discrete ACO the uncertainty in the estimation of FS was large and many fitness function evaluations were needed to arrive to an optimum value. In ACO, the number of fitness evaluations equals to product of population size with the total number of iterations as in each iteration the fitness function is evaluated only once. As different algorithms use different amounts of consumption of fitness function evaluations per iteration so a comparison made only on maximum number of iterations as a stop condition is not justified $[43,44]$.

In this study, an extended form of ACO, known as continuous ant colony optimisation $\left(\mathrm{ACO}_{R}\right)$, capable to solve problems in the continuous domain, is used for computing the factor of safety for slope stability calculations. The minimum factor of safety is identified using $\mathrm{ACO}_{R}$ with control parameters varying continuously, differently than previously used approaches of ACO working with discrete intervals. Furthermore, the $\mathrm{ACO}_{R}$ is validated against theoretical benchmark example. Additionally, an actual field failure is back-analysed using this technique. We will show that results obtained by the proposed technique are in agreement with those of other researches and with those obtained by standard software.

Apart from being applied in slope stability, ACO and its variants have been used in other civil engineering optimisation problems, e.g. identifying structural damages from changes in natural frequencies [45-47], allocation of railway platforms [48], design of concrete retaining walls [49], damage detection in structures [50], guiding vehicles to less congested paths [51,52], forecasting river flow [53] to mention a few of them. These works demonstrate the potential of ACO techniques in solving civil engineering optimisation problems.

\section{Setting of slope stability analysis}

For setting up a slope stability analysis, the first step is to generate a kinematically feasible slip surface for the slope and calculate the factor of safety associated with it. The study considered a non-circular slip surface by linking straight lines using a method proposed by Cheng [54]. To start the generation of a slip surface, the failure mass is divided into $\mathrm{N}$ slices of equal width as shown in Fig. 1 . The limits of variable $x_{1}$ and $x_{N+1}$ are the entry $\left(x_{l}, x_{u}\right)$ and exit points range $\left(x_{L}, x_{U}\right)$ (Fig. 1) which depend on the experience of the practitioner while all other control variables $\left(\sigma_{1}, \ldots, \sigma_{N-1}\right)$ vary between 0 and 1 . The slip surface can hence be described as follows:

$$
V=\left[V_{1}, V_{2}, \ldots, V_{N}, V_{N+1}\right]
$$

In terms of coordinates it can be written as:

$$
V=\left[\left(x_{1}, y_{1}\right),\left(x_{2}, y_{2}\right), \ldots,\left(x_{N}, y_{N}\right),\left(x_{N+1}, y_{N+1}\right)\right]
$$

The $\mathrm{x}$ co-ordinates of points lying between entry and exit $\left(x_{2}, \ldots, x_{N}\right)$ are equally spaced as follows: 


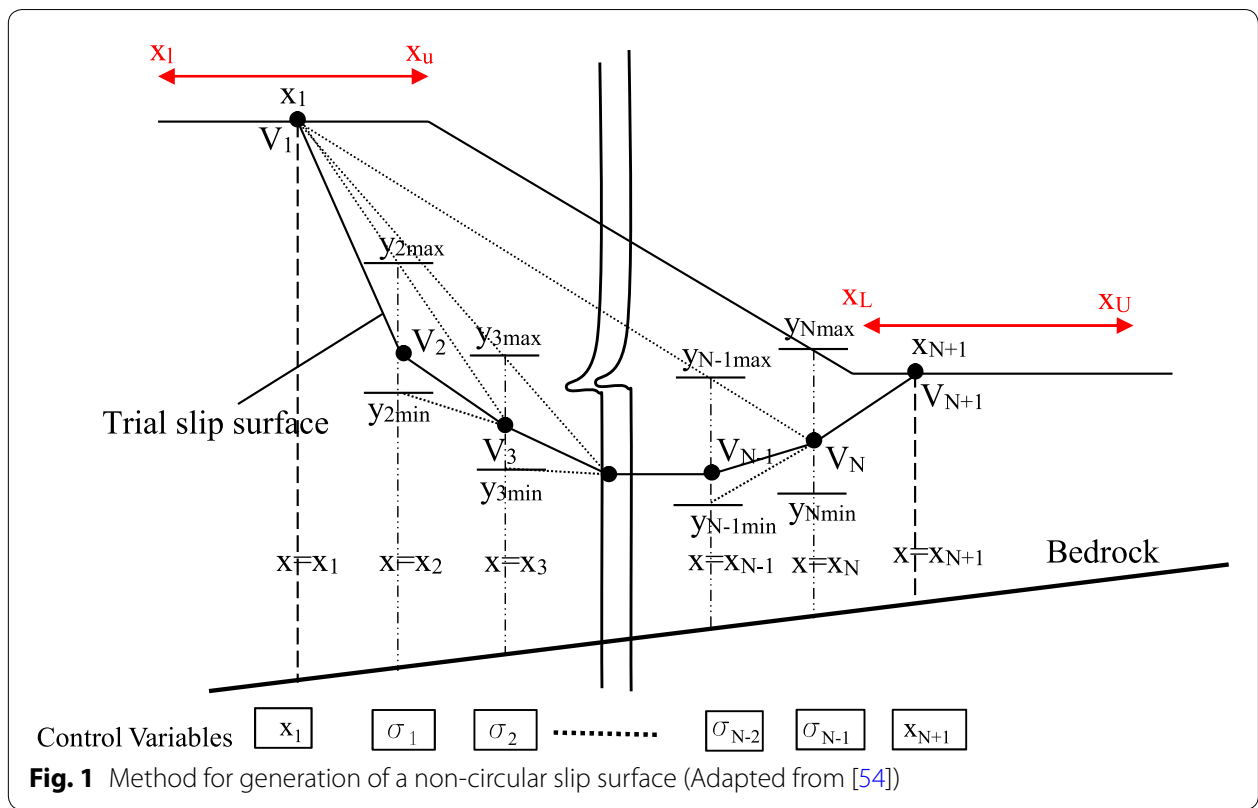

$$
x_{i}=x_{1}+\left(\frac{x_{N+1}-x_{1}}{N}\right) \times(i-1) ; \quad i=2,3, \ldots N
$$

For determining the $y$-coordinates of each vertex $\left(V_{2}, \ldots, V_{N}\right)$ the upper and lower bounds ( $y_{\text {imax }}$ and $\left.y_{\text {imin }}\right)$ are considered, where i denotes the index of vertices. The process of determination of upper and lower bounds of $y_{i}$ is shown in Fig. 1. As can be seen $y$-coordinates of each vertex are different with each slip trial surface, so control variable $\sigma$ is used which always varies between 0 to 1 irrespective of the considered slip surface. Hence, $y_{i}$ can be determined dynamically between the maximum and minimum values for $y_{i}$ by the following equation:

$$
y_{i}=y_{\text {imin }}+\left(y_{\text {imax }}-y_{\text {imin }}\right) \times \sigma_{i-1} ; \quad i=2, \ldots, N
$$

The optimisation problem for searching a critical slip surface can be summarised as:

$$
\min f(x \leftarrow \mathbf{V}) \text { s.t } x_{l} \leq x_{1} \leq x_{u} ; x_{L} \leq x_{N+1} \leq x_{U} ; 0<\sigma_{i}<1 i=1, \ldots, N-1
$$

where $\mathbf{V}$ can be obtained by the procedure explained above.

A generalised slip surface is thus defined by a set of $N+1$ decision variables to optimise; $x_{1}, \sigma_{1}, \ldots, \sigma_{N-1}, x_{N+1}$. Each set of combinations of decision variables represents one trial slip surface.

After the generation of a trial slip surface, the second step is to determine the factor of safety (fitness function) associated with each slip surface. The original formulation of Morgenstern-Price method for calculating FS of a slope involves nonlinear equations which have to be solved numerically. The algorithm developed by Zhu et al. [55] based on the Morgenstern-Price method is used here to calculate the factor of safety. Finally, all the control variables $\left[x_{1}, \sigma_{1}, \ldots, \sigma_{N-1}, x_{N+1}\right]$ are optimised iteratively until 
the termination criteria is met using the $\mathrm{ACO}_{R}$ algorithm to find the right combination leading to the minimum safety factor.

\section{Theoretical background of ant colony optimisation (ACO)}

\section{Ant colony optimisation (ACO)}

Ant colony optimisation proposed by Dorigo and Stutzle [56] is a biologically inspired swarm optimisation method mimicking the foraging behaviour of ant colonies. They find the shortest possible path from their origin point (start) to the food source (finish) by releasing a chemical known as pheromone. When a lone ant randomly tries to find the food source, it can sense the pheromone trail left by other ants that have previously followed the same path. The pheromone trail stores local information of the path and is reinforced as each successive ant passes. It is a population based search technique for optimisation of problems having many inputs based on trail-laying and trail-following of its members to find the optimum solution. This foraging behaviour can be artificially simulated and can be used to solve engineering problems.

The research presented here explores the ACO optimisation algorithm in calculating the minimum factor of safety of the slope. In fact, the problem of finding the critical failure surface is very much similar to problems leading to a shortest optimised path. Figure 2 shows the permitted paths for a number of variables with their limits. The objective is to find the path which leads to a minimum factor of safety. The weighted connected graph shows the set of nodes and connections between them. In each case, a node represents the value of the variable to be chosen. The ants move from node to node to the right recording each node visited so as to update the pheromone trail. The lines between the nodes indicate the flow of ant path from one node to the next, chosen probabilistically as explained in the following point 1 on probabilistic transition and depending on the intensity of pheromone on that node. When ACO framework is implemented as an algorithm for solving either discrete or continuous optimisation problem, the following rules have to be followed by ants:

\section{Probabilistic transition:}

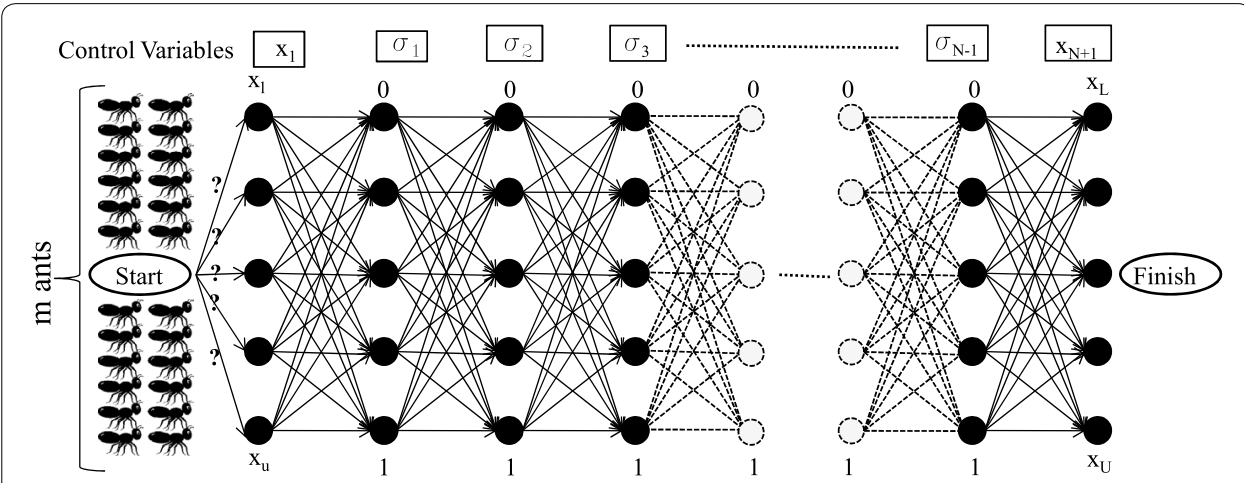

Fig. 2 Architecture of the discrete ACO algorithm and construction of search space for $m$ number of ants for optimisation of slope stability analysis $\left(x_{1}, \sigma_{1}, \ldots, \sigma_{N-1}, x_{N+1}\right.$ are control variables with their limits for FS computation as shown in Fig. 1) 


$$
p_{i j}^{k}= \begin{cases}\frac{\tau_{i j}^{\alpha}}{\sum_{l \in N_{i}^{k} \tau_{i j}^{\alpha}}}, \quad \forall j \in N_{i}^{k}, i=1,2, \ldots, N+1, j=1,2, \ldots, m \\ 0 \quad \text { otherwise }\end{cases}
$$

where $p_{i j}^{k}$ is transition probability for ant $k$ to choose node $\mathrm{j}$ when located at node $\mathrm{i}$, $\tau_{i, j}$ is the amount of pheromone on trail associated with node $(i, j), N_{i}^{k}$ is the possible neighbourhood nodes of ant $k$ when placed at node $i$ and $\alpha>0$ is a parameter denoting the degree of importance of the pheromones. $\alpha$ is generally chosen as 1 [56] to allow diversification in solutions otherwise a higher value of $\alpha$ will influence ACO to intensify amount of pheromone to previously visited nodes leading to premature convergence.

The rule as shown in Fig. 3 explains how the ant will select the path with a higher level of pheromone concentration. The ant path is characterised by vector $x_{1,2} \rightarrow \sigma_{1,3} \rightarrow \sigma_{2,1} \rightarrow \sigma_{i, 4} \rightarrow x_{N+1,5}$ as this path has maximum pheromone concentration at these nodes (marked by solid circles). In essence, calculation in this case is carried out starting from the first control variable $\left(x_{1}\right)$ and exhaustively identifying all possible paths until reaching the finish variable $\left(x_{N+1}\right)$. The movement to successor node is determined by the stochastic decision rule given in Eq. 6 which is function of its local pheromone level and relative importance of pheromone.

2. Updating pheromone concentration:

$$
\tau_{i j}^{u p} \leftarrow(1-\rho) \times \tau_{i j}^{p r e v}+\Delta \tau_{i j}^{k}
$$

where $\rho$ is the evaporation rate varying from 0 to 1 of pheromone and controls the quantity of pheromone on each trail path and $\Delta \tau_{i j}^{k}$ is the additional pheromone

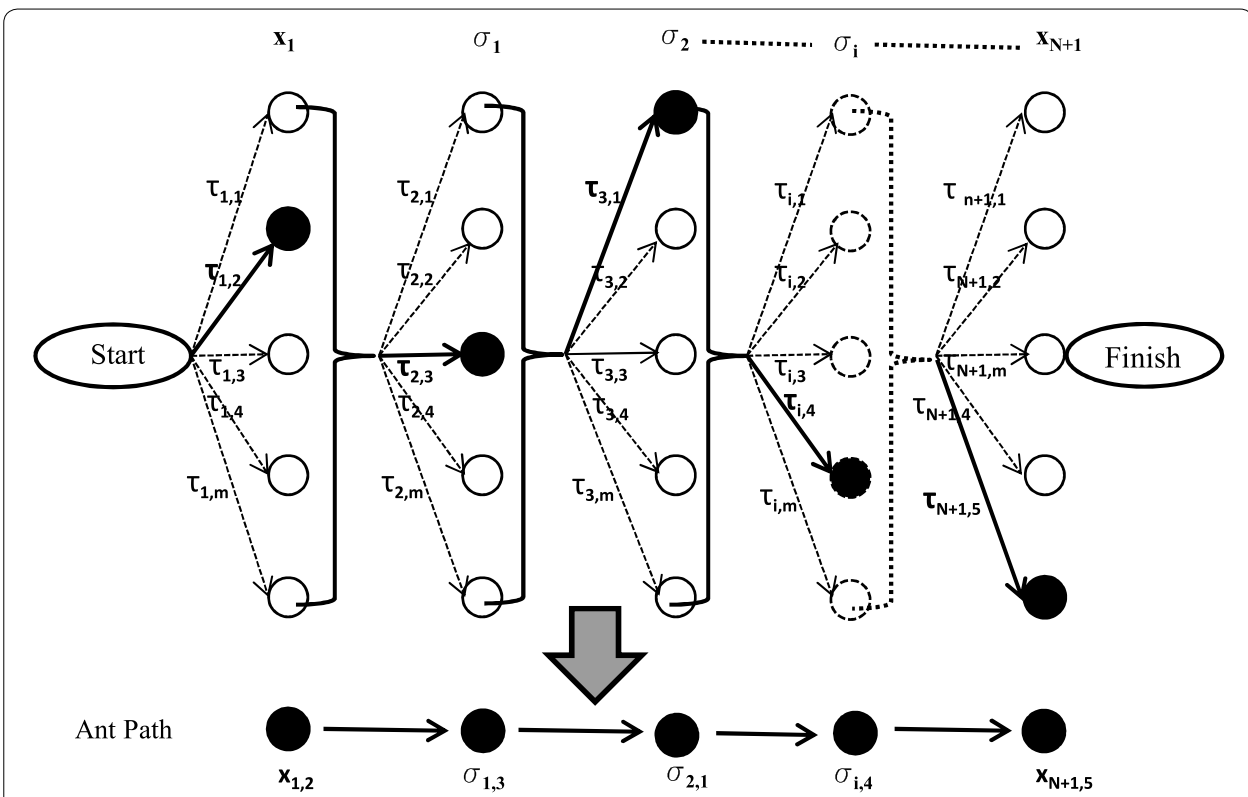

Fig. 3 Selection process of possible ant paths as a function of pheromone concentration level $\left(\tau_{i j}\right)$. The solid nodes representing high pheromone concentration will have more probability of selection than their counterparts 
deposited by $k$ th ant at node $(i, j) . \tau_{i j}^{u p}$ and $\tau_{i j}^{p r e v}$ denotes the updated and previous pheromone trail at node $(i, j)$. A higher value of $\rho$ will reduce the number of significant iterations, this decreasing the diversification of solutions leading to premature convergence.

For discrete ant colony optimisation algorithm, a number of artificial ants are considered (m). Each ant builds a solution by visiting node to node on the graph (Fig. 3) in forward direction where each node has transition probability attached to it. An ant visits nodes representing variables of the optimisation problem $(1$ to $N+1)$. The initial intensity of pheromone on each node can be initialised to 1 , so that all the paths have an equal probability of being chosen and no path is being left out. Hence, at first iteration, all the nodes have the same pheromone concentration that is $\tau_{i j}=\tau_{0}=1(\mathrm{i}=1,2, \ldots, \mathrm{N}+1 ; \mathrm{j}$ $=1,2, \ldots, \mathrm{m})$. The pheromone concentration at each node is globally updated dynamically in each iteration after all ants have successfully deposited the pheromone on their trail path. The pheromone updating aims to boost the pheromone trails in promising regions. The shorter path will have a higher quantity of pheromone level than the longer path owing to its lower evaporation. The iterations are carried out for a number of cycles to produce the optimum solution. At the end of iteration cycle, based on the quality of solution constructed by ants, more pheromone will be concentrated on nodes that construct the best solution.

\section{ACO for continuous optimisation- $\mathrm{ACO}_{R}$}

An extension of the original ACO algorithm developed for discrete domains is continuous ant colony optimisation $\left(\mathrm{ACO}_{R}\right)$ which works for decision variables in continuous domains. The $\mathrm{ACO}_{R}$ has been more practical to use as in practical applications decision variables lay in continuous domains. The continuous ACO algorithm was proposed by Socha et al. [57] inspired from discrete ACO and works without making any major changes in the original ACO framework. The fundamental idea of $\mathrm{ACO}_{R}$ is to consider continuous probability density function (PDF) $P(x)$ instead of discrete PDF according to Eq. 6 to construct solutions. As the domain of each variable in $\mathrm{ACO}_{R}$ is continuous, an ant sampling is based on a continuous probability-density function $P(x)$. The procedure of using $\mathrm{ACO}_{R}$ meta-heuristics is outlined below.

In this study, slip surface is represented by a set of $N+1$ decision variables; $x_{1}, \sigma_{1}$ ,..., $\sigma_{N-1}, x_{N+1}$. For a $N+1$ dimensional continuous problem, an archive of $k$ solutions denoted by $S$ (Eq. 8 ) is constructed. Each solution in the archive contains the value of $\mathrm{N}+1$ variables used to construct a generic trial slip surface and the factor of safety associated with it. This solution archive is equivalent to pheromone model used by discrete ACO to keep a memory of the search history. As pheromone cannot be updated directly, the equivalent step is updating the solution archive. The size of the archive $k$, which is also a controlling parameter of the algorithm, must be greater than the dimensions of variables being solved. For example, in our case we are using 30 slices $(N)$ for slip surface discretisation having $31(N+1)$ control variables, so a minimum archive size of 31 must be taken. The structure of solution archive in $\mathrm{ACO}_{R}$ comprises of three matrices as follows.

The position of control variables defining slip surface outlined in "Setting of slope stability analysis" section are stored in the following matrix $S$ of size $k \times N+1$ : 


$$
S=\left(\begin{array}{ccccccc}
1 & 2 & 3 & & i & N & N+1 \\
x_{1}^{1} & \sigma_{1}^{1} & \ldots & \ldots & \ldots & \sigma_{1}^{N-1} & x_{1}^{N+1} \\
x_{2}^{1} & \sigma_{2}^{1} & \ldots & \ldots & \ldots & \sigma_{2}^{N-1} & x_{2}^{N+1} \\
\vdots & \vdots & \vdots & \vdots & \vdots & \vdots & \vdots \\
x_{j}^{1} & \sigma_{j}^{1} & \ldots & \ldots & & \sigma_{j}^{N-1} & x_{j}^{N+1} \\
\vdots & \vdots & \vdots & \vdots & \vdots & \vdots & \vdots \\
x_{k}^{1} & \sigma_{k}^{1} & \ldots & \ldots & \ldots & \sigma_{k}^{N-1} & x_{k}^{N+1}
\end{array}\right)_{k}
$$

The matrix $S$ represents $k$ trial slip surfaces used in $\mathrm{ACO}_{R}$ solution archive. For a more general case matrix $S$ of size $k \times N+1$ can be written as

$$
S=\left(\begin{array}{ccccccc}
1 & 2 & 3 & & i & \multicolumn{2}{c}{N+1} \\
s_{1}^{1} & s_{1}^{2} & s_{1}^{3} & \ldots & s_{1}^{i} & \ldots & s_{1}^{N+1} \\
s_{2}^{1} & s_{2}^{2} & s_{2}^{3} & \ldots & s_{2}^{i} & \ldots & s_{2}^{N+1} \\
\vdots & \vdots & \vdots & \vdots & \vdots & \vdots & \vdots \\
s_{j}^{1} & s_{j}^{2} & s_{j}^{3} & \ldots & s_{j}^{i} & \ldots & s_{j}^{N+1} \\
\vdots & \vdots & \vdots & \vdots & \vdots & \vdots & \vdots \\
s_{k}^{1} & s_{k}^{2} & s_{k}^{3} & \ldots & s_{k}^{i} & \ldots & s_{k}^{N+1}
\end{array}\right)_{k}
$$

where $s_{j}^{i}$ denotes the value of $j$ th solution of the $i$ th unknown variable. The movement of ants along the path leads to different slip surfaces, with a set of variables stored in matrix $S$.

$f$ is the matrix storing the factor of safety of each slip surface:

$$
f=\left(\begin{array}{c}
f\left(x_{1}^{1}, \sigma_{1}^{1} \ldots, \sigma_{1}^{N-1}, x_{1}^{N+1}\right) \\
f\left(x_{2}^{1}, \sigma_{2}^{1} \ldots, \sigma_{2}^{N-1}, x_{2}^{N+1}\right) \\
\vdots \\
f\left(x_{j}^{1}, \sigma_{j}^{1} \ldots, \sigma_{j}^{N-1}, x_{j}^{N+1}\right) \\
\vdots \\
\vdots \\
f\left(x_{k}^{1}, \sigma_{k}^{1} \ldots, \sigma_{k}^{N-1}, x_{k}^{N+1}\right)
\end{array}\right)
$$

$\omega$ is the matrix saving the associated weights with each solution of the archive: 


$$
\omega=\left(\begin{array}{c|c}
\omega_{1} & 1 \\
\omega_{2} & 2 \\
\vdots & \vdots \\
\omega_{j} & j \\
\vdots & \vdots \\
\omega_{k} & k
\end{array}\right.
$$

To initialise the algorithm, random sets of all $k$ solutions having $N+1$ control variables are chosen within the specified parameter range to construct all the rows of the archive matrix $S$, which is equivalent to pheromone initialisation in discrete ACO algorithm. The solutions in the archive are always sorted according to fitness function $\left(f_{1} \leq f_{2} \leq \ldots \leq\right.$ $f_{k}$ ) values. In the following examples, the safety factor associated with each slip surface is computed by Spencer's method, i.e. Morgernester-Price method with interslice function $f(x)=1$.

As the iteration proceeds, better sets of solutions are appended to the population and solutions with lower fitness values are removed from it. At each iteration, $m$ new solutions are produced and among them the best $k$ solutions are retained. The step is basically biased towards selecting best solutions and is equivalent to pheromone updating in discrete ACO. Mathematically for construction a solution as shown in matrix $S$, an ant has to choose the decision variable $s_{j}^{i}$ for every unknown variable $i=$ $1,2, \ldots, N+1$. The row $j$ of matrix $S$ is chosen probabilistically for all $N+1$ construction steps to generate new solutions for the archive as follows:

$$
p_{j}=\frac{\omega_{j}}{\sum_{l=1}^{k} \omega_{l}} \forall j=1,2, \ldots, k
$$

where $\omega$ is defined as matrix of associated weights of each solution whose elements are computed by employing a gaussian function [57]. For the jth row of the archive associated weight $\omega_{j}$ is defined as follows:

$$
\omega_{j}=\frac{1}{q k \sqrt{2 \pi}} e^{-\frac{(j-1)^{2}}{2(q k)^{2}}}
$$

where $q$ is the selection pressure varying between 0 to 1 of the algorithm which controls the exploration and exploitation rate in $\mathrm{ACO}_{R}$. If $q$ is small, the best-ranked solutions are preferred, while for larger values of $q$, the probability of choosing control variables becomes more uniform.

The ant subsequently chooses the value $s_{j}^{i}$ and samples its neighbourhood for a new value for variable $i=1,2, \ldots, \mathrm{N}, \mathrm{N}+1$ and reiterates this operation for all control variables using the same $j$ th solution using a probability density function (PDF). Although different choices are possible, in this work a Gaussian function is again chosen [6]: 


$$
P(x)=\frac{1}{\sigma_{j}^{i} \sqrt{2 \pi}} e^{-\frac{\left(x-\mu_{j}^{i}\right)^{2}}{2\left(\sigma_{j}^{i}\right)^{2}}}
$$

where for the mean $\mu_{j}^{i}$, the values of the $i$ th variable for all the solutions deposited the archive $S$ become the elements of the vector $\mu_{j}^{i}$ :

$$
\mu_{j}^{i}=\left\{\mu_{1}^{i}, \mu_{2}^{i}, \ldots, \mu_{k}^{i}\right\}=\left\{s_{1}^{i}, s_{2}^{i}, \ldots, s_{k}^{i}\right\}
$$

Furthermore, the values of standard deviation $\sigma_{j}^{i}$ can be calculated dynamically as the average distance from the chosen solution $s_{j}^{i}$ to other solutions and then multiplying with the parameter $\xi>0$ which determines the convergence speed of the algorithm as follows:

$$
\sigma_{j}^{i}=\xi \sum_{r=1}^{k} \frac{\left|s_{r}^{i}-s_{j}^{i}\right|}{k-1}
$$

After each iteration, the set of newly generated solutions will be refreshed in the archive, and then the archive for keeping the best $k$ solutions probabilistically. The candidate solutions stored in archive are used to modify the probability distribution such that it is biased towards sampling high quality solutions. This process is repeated until the maximum number of iterations are reached.

\section{Application of the proposed technique}

For the purpose of demonstrating the efficacy of the $\mathrm{ACO}_{R}$ algorithm to locate the critical slip surface and the associated minimum safety factor, two case studies are analysed. One case study is from published literature and the second one is from a failed road embankment located in eastern India. Since ACO is a non-deterministic method i.e. giving different solution for each computation, the FS values and standard deviation reported are based on a number of simulations equal to 10 to capture the uniformity in the solutions.

As described in "Theoretical background of ant colony optimisation (ACO)" section, in the $\mathrm{ACO}_{R}$ introduction, the size of the archive $(k)$, the number of ants $(m)$, the selection pressure $(q)$ and pheromone evaporation rate $(\xi)$ play important role in convergence speed of the algorithm. To strike a balance between diversification and convergence of the search process, proper selection of these parameters is important. After carrying out parametric analysis and following the suggestions by Socha and Dorigo [57] and Majumdar et al. [58], the constant parameters of the proposed algorithm have been set up as follows: $m=50, q=0.5, \xi=1, k=40$ and maximum iterations $=200$.

\section{Literature example}

The literature example with four soil layers has been taken from Zolfaghari et al. [29]. Geometry of the soil slope and soil properties are shown in Fig. 4. The soil layers are horizontal except for the first and second ones which are slightly inclined. The example is analysed using $\mathrm{ACO}_{R}$ in dry conditions for two cases: (1) No earthquake loading (case 1), (2) Earthquake loading (pseudo-static horizontal coefficient of 0.1) (case 2). 
Table 1 Comparison of factors of safety obtained for literature example with different optimisation methods for case 1 (no earthquake loading) and case 2 (earthquake loading)

\begin{tabular}{lll}
\hline Source & Optimisation method & FS \\
\hline Zolfaghari et al. (case 1) [29] & Genetic algorithm (GA) & 1.48 \\
Zolfaghari et al. (case 2) [29] & Genetic algorithm (GA) & 1.37 \\
Cheng et al. (case 1) [20] & Particle swarm optimisation (PSO) & 1.3323 \\
Cheng et al. (case 2) [20] & Particle swarm optimisation (PSO) & 1.047 \\
Cheng et al. (case 1) [20] & Modified particle swarm optimisation (MPSO) & 1.349 \\
Cheng et al. (case 2) [20] & Modified particle swarm optimisation (MPSO) & 1.059 \\
Kahatadeniya et al. (case 1) [17] & Ant colony optimisation (ACO) & 1.501 \\
Kahatadeniya et al. (case 2) [17] & Ant colony optimisation (ACO) & 1.091 \\
Kang et al. (case 1) [23] & Artificial bee colony (ABC) & 1.292 \\
Geoslope (case 1) [35] & Optimise function using Monte Carlo random walk & 1.333 \\
Geoslope (case 2) [35] method based on [16, 40] & 1.047 \\
ACO $_{R}$ (500 Iterations) (case 1) & Optimise function using Monte Carlo random walk & \\
ACO $R_{R}$ (500 Iterations) (case 2) & method based on [16, 40] & $1.293-1.316$ \\
\hline
\end{tabular}

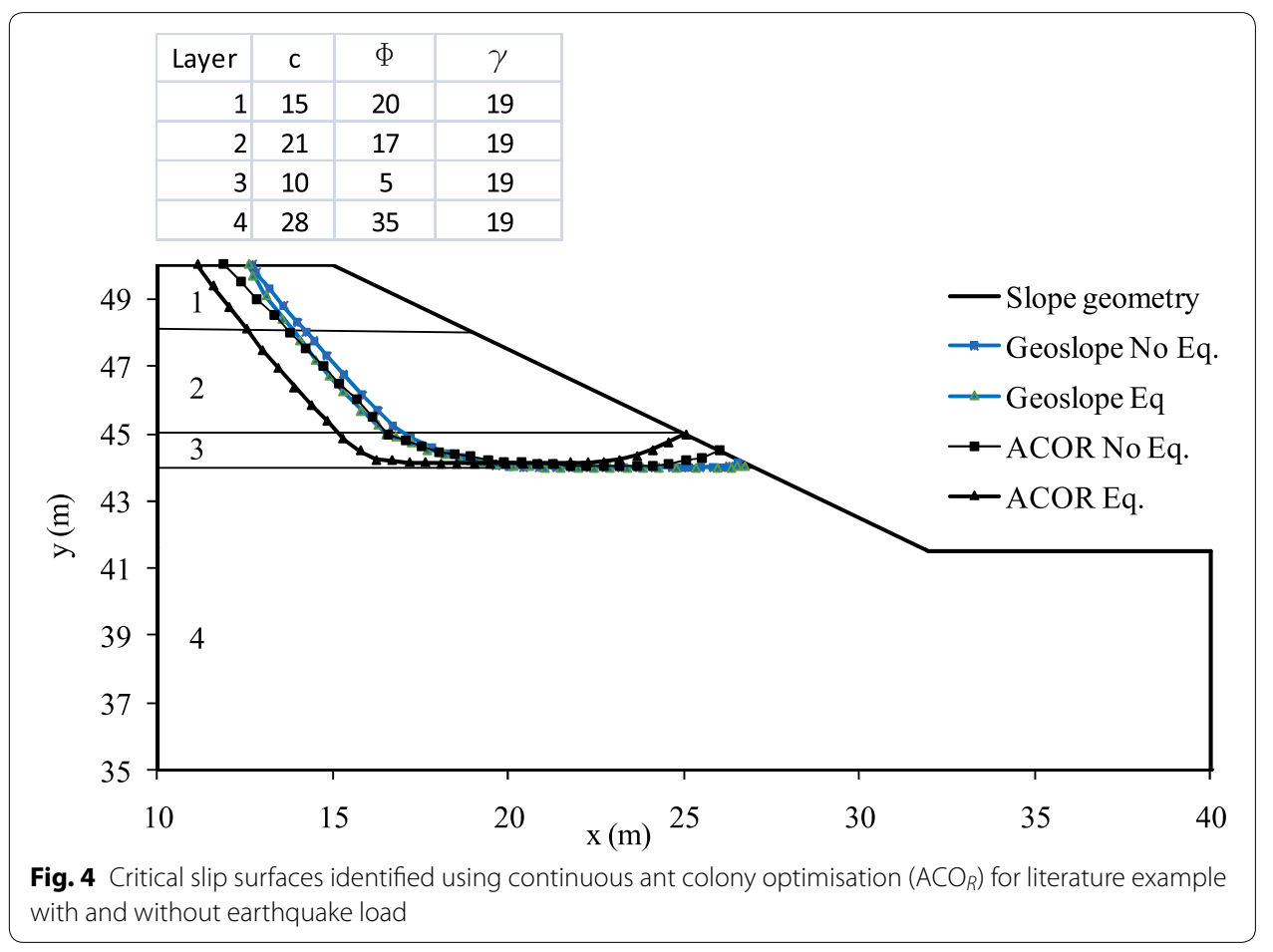

Table 1 summarises the results of both loading cases and compares them with those of optimisation techniques used by other researchers. Figure 4 reports the critical slip surface obtained and the one determined by the commercial software Geoslope [35]. The minimum FS obtained for case 1 i.e without earthquake loading is 1.293 with standard deviation of 0.008 and 1.010 in the presence of earthquake loading, with a standard deviation of 0.025 . The proposed algorithm gives lower factors of safety than the GA, 
PSO and $\mathrm{ABC}$ for the two cases considered. Comparative assessment shows that the FS computed by MPSO, ACO and Geoslope analysis tend to be similar to that computed by the proposed approach, showing the efficacy of this latter. The lower FS represents the capture of global minimum.

However, computational experiments indicate that, for practical purposes, the desired accuracy can be achieved after 200 iterations (i.e., after a number of 10,000 fitness function evaluations), and thus, it is computationally similar to $\mathrm{ABC}$. The fitness function evaluations when compared to discrete ACO $(150,000)$ [17] are significantly reduced, thereby leading to reduction in computation time. Furthermore, the computational time, determined by calculating the average time for 10 computations, was assessed on the basis of an Intel(R) Core(TM) i5-7500 CPU 3.40 GHz processor with 4.00 GB RAM. For one simulation, average computing time is approximately $40 \mathrm{~s}$ for the $\mathrm{ACO}_{R}$ algorithm.

\section{Engineering application}

The proposed methodology is also applied to a case study of an embankment failure, located in eastern India. The National Highway no. 316 is a very important highway in eastern India and a part of it connects the cities of Bhubaneswar and Puri (Fig. 5a) is constructed over soft clay deposits. The existing two lane national highway is being augmented in capacity by increasing the number of lanes. Furthermore, a rail over bridge was proposed in the place of barrier crossing. To take the road to the elevation of the rail over bridge, an approach embankment was proposed with a maximum height of $11 \mathrm{~m}$. The embankment is $27 \mathrm{~m}$ wide at the top and $35 \mathrm{~m}$ at the bottom with a side slope of $70^{\circ}$. During construction, signs of distress were observed on the left hand side of the embankment, when the embankment height reached its maximum value. After

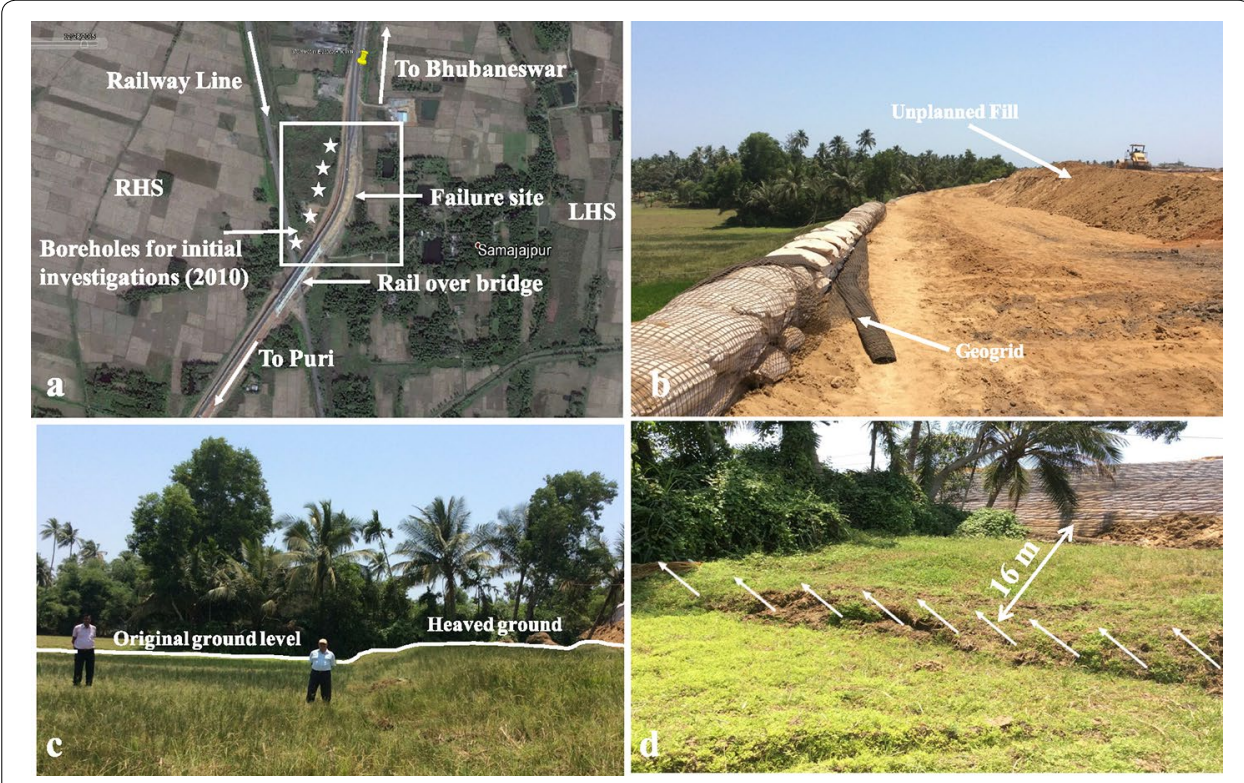

Fig. 5 a Google image of embankment failure site in 2015 with the location of boreholes for initial investigation; $\mathbf{b}$ top of the embankment with unplanned fill and geogrid; $\mathbf{c}, \mathbf{d}$ Heave location at about $16 \mathrm{~m}$ from toe 
2 months, the approach embankment failed (Fig. 5c, d) at Samajajpur at about $12.8 \mathrm{~km}$ from the city of Puri.

\section{Geotechnical investigations}

Five boreholes concentrated primarily on the right hand side were drilled for the initial investigations. After failure, 23 boreholes were drilled both on the left hand and right hand side (Fig. 5a). The construction was stopped as heaving was seen at about $16 \mathrm{~m}$ from the embankment (see Fig. 5b-d). The subsoil was throughly characterised for index properties and undrained shear strength of the foundation soil. Figure 6 shows the variation of undrained shear strength as obtained from initial and post failure investigations, as well as their respective average profiles. It can be observed that the left hand side soil profile is weaker than the soil profile in right hand side. Average values of soil strength parameters were used in the analysis for both the cases considered (first and second investigation) obtaining the results reported in Fig. 7.

\section{Analysis with $\mathrm{ACO}_{R}$}

In this case, the algorithm was modified as the entry point $x_{1}$ is not variable and is taken as the middle point of the road $\left(x_{1}=0\right)$. Indeed, the pictures of the site after the failure reveal that the entry of the failure surface was in the middle of the embankment. The factor of safety was calculated using Slope/w [35], a Limit Equilibrium Method software using Morgenstern Price method and a Finite Element Method using strength reduction module in RS2 [37]. Table 2 compares the results from the analysis, and it can be seen

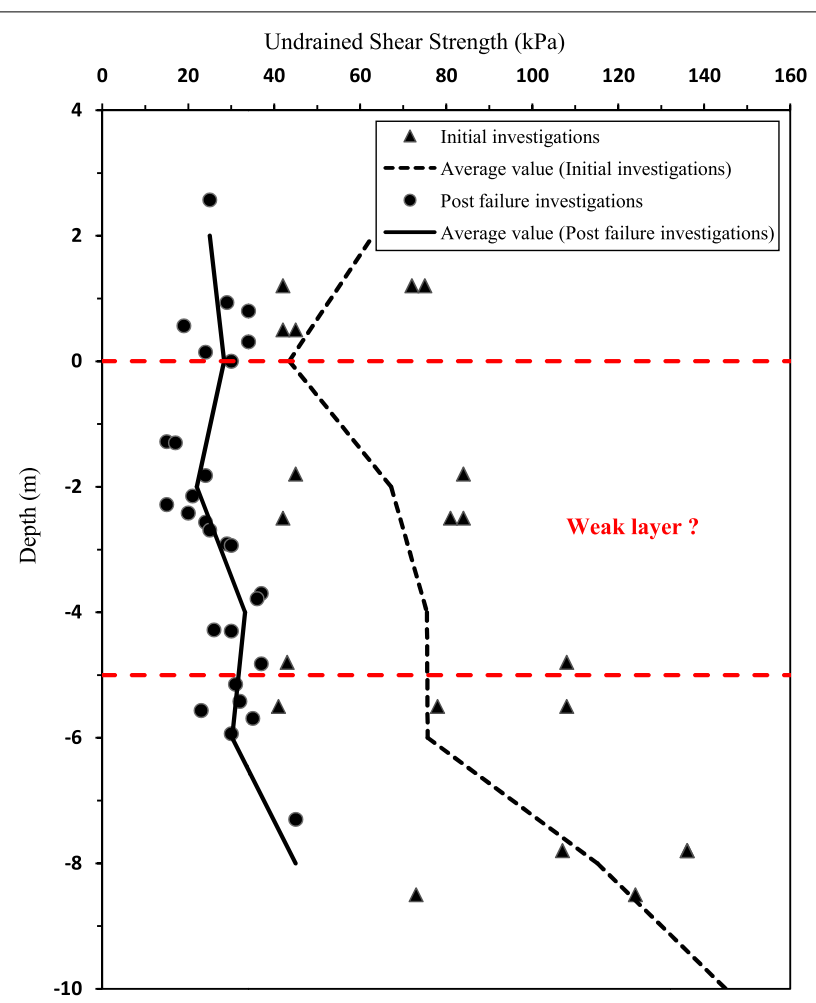

Fig. 6 Undrained shear strength with depth based on initial and post-failure investigations 


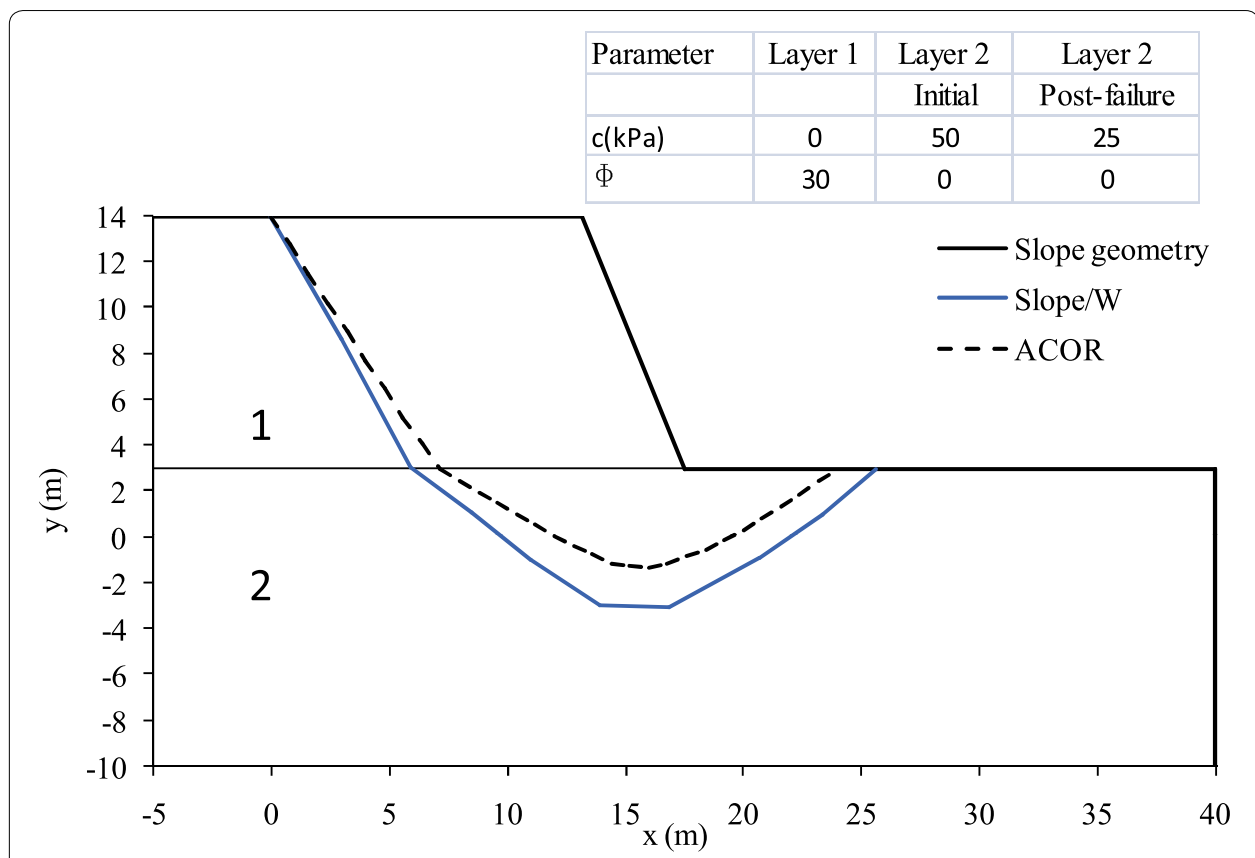

Fig. 7 Critical slip surface identified using $\mathrm{ACO}_{R}$ for the failed slope by considering the parameters resulting from post-failure investigations

\begin{tabular}{|c|c|c|c|}
\hline Investigations & LEM-MP & FE-SRM & $\mathrm{ACO}_{R}$ \\
\hline Initial & 1.19 & 1.15 & $1.098-1.132$ \\
\hline Final & 0.68 & 0.637 & $0.617-0.639$ \\
\hline
\end{tabular}

they closely match with each other. The $\mathrm{ACO}_{R}$ analysis provides a FS range whose lower value is from 3 to $9 \%$ lower than the FS of the other analyses and whose upper value has a difference of $6 \%$ at the most from the other analyses.

It can be concluded that FS is more than 1 if we consider the results of the initial investigation (as designers did) with LEM, FEM and ACO whereas FS $<1$ if we consider the results of the investigation which was carried out after the failure of the embankment. Figure 7 reports the critical slip surface obtained by the calculation carried out using $\mathrm{ACO}_{R}$, obtaining the safety factors reported in Table 2 . The effect of geogrids and pore water pressure was not considered in the analysis for simplification but could be incorporated with minor modification in the Matlab sub-routines.

\section{Summary and conclusions}

In this paper, we presented a technique for slope stability assessment using continuous ant colony optimisation algorithm $\left(\mathrm{ACO}_{R}\right)$. The algorithm is tested with literature benchmark example and with data of landslide failure coming from slope failure at a road embankment site. The parameters used in the $\mathrm{ACO}_{R}$ algorithm are calibrated to avoid trapping into local minima leading to sub-optimal results. We also presented an 
application of our technique applied to one heterogeneous slope having one weak soil layer. The results of the proposed technique are compared with reported results of GA, $\mathrm{PSO}, \mathrm{HS}, \mathrm{ABC}$ and discrete ACO for the chosen literature example. The performance of $\mathrm{ACO}_{R}$ was found to be better than that of the other approaches in terms of computing the minimum FS and determining the critical slip surface. Such characteristics make the proposed algorithm suitable for practitioners to identify the critical non-circular slip surface in real slope stability problems.

\section{Acknowledgements}

The authors would like to acknowledge M/S Z-Tech for providing us the results of in-situ and laboratory investigation on the embankment in Samajajpur.

\section{Authors' contributions}

MM and MSB carried out the slope stability analysis for the failed embankment. GVR assisted in carrying out the in-situ and laboratory investigation on the embankment site. RV contributed to conceptualization, draft preparation, writing review and editing. All authors read and approved the final manuscript.

\section{Competing interests}

The authors declare that they have no competing interests. All the authors have the read the manuscript and have full consent in submitting it.

\section{Author details}

${ }^{1}$ School of Infrastructure, Indian Institute of Technology Bhubaneswar, Argul, Khordha 752050, India. ${ }^{2}$ Civil and Environmental Engineering, University of California, Davis, USA. ${ }^{3}$ Department of Civil Engineering, Indian Institute of Technology, Delhi 110016, India. ${ }^{4}$ Scuola di Ingegneria, Università degli Studi della Basilicata, Potenza, Italy.

Received: 26 September 2019 Accepted: 18 March 2020

Published online: 27 March 2020

\section{References}

1. Bishop AW (1955) The use of the slip circle in the stability analysis of slope. Geotechnique 6(1):7-17

2. Janbu N (1954) Application of composite slip surface for stability analysis. In: European conference on stability of earth slopes, Stockholm vol 3, pp 43-49

3. Morgenstern NR, Price VE (1965) The analysis of the stability of general slip surfaces. Geotechnique 15(1):79-93

4. Viggiani C (1981) Ultimate lateral load on piles used to stabilize landslides. In: Proceedings of the 10th international conference on soil mechanics and foundation engineering, Vol 3, Balkema, Rotterdam, the Netherlands, pp 555-560

5. Fast Lagrangian Analysis of Continua (FLAC), version 5.0 (2005) ITASCA Consulting Group, Inc., Minneapolis

6. Soren K, Budi G, Sen P (2014) Stability analysis of open pit slope by finite difference method. Int J Res Eng Technol 3:326-334

7. Saeed MS, Maarefvand P, Yaaghubi E (2015) Two and three-dimensional slope stability analyses of final wall for miduk mine. Int J Geo-Eng 6(1):9

8. Duncan JM (1996) State of the art: limit equilibrium and finite-element analysis of slopes. J Geotech Eng 122(7):577-596

9. Griffiths DV, Lane PA (1999) Slope stability analysis by finite elements. Géotechnique 49(3):387-403

10. Zheng H, Sun G, Liu D (2009) A practical procedure for searching critical slip surfaces of slopes based on the strength reduction technique. Comput Geotech 36(1):1-5

11. Cheng YM, Lansivaara T, Wei WB (2007) Two-dimensional slope stability analysis by limit equilibrium and strength reduction methods. Comput Geotech 34(3):137-150

12. Liu SY, Shao LT, Li HJ (2015) Slope stability analysis using the limit equilibrium method and two finite element methods. Comput Geotech 63:291-298

13. Park B-S, Cho H, Youn S-P, Park D-I (2016) Analysis and evaluation of stability for the reactivated landslide along deep-seated weakness zones. Int J Geo-Eng 7(1):3

14. Baker R (1980) Determination of the critical slip surface in slope stability computations. Int J Numer Anal Methods Geomech 4(4):333-359

15. Bardet JP, Kapuskar MM (1989) A simplex analysis of slope stability. Comput Geotech 8(4):329-348

16. Greco VR (1996) Efficient Monte Carlo technique for locating critical slip surface. J Geotech Eng 122(7):517-525

17. Kahatadeniya KS, Nanakorn P, Neaupane KM (2009) Determination of the critical failure surface for slope stability analysis using ant colony optimization. Eng Geol 108(1):133-141

18. Gao W (2016) Premium-penalty ant colony optimization and its application in slope stability analysis. Appl Soft Comput 43:480-488

19. Gao W (2016) Determination of the noncircular critical slip surface in slope stability analysis by meeting ant colony optimization. J Comput Civil Eng. https://doi.org/10.1061/(ASCE)CP.1943-5487.0000475

20. Cheng YM, Li L, Chi SC, Wei WB (2007) Particle swarm optimization algorithm for the location of the critical noncircular failure surface in two-dimensional slope stability analysis. Comput Geotech 34(2):92-103

21. Li YC, Chen YM, Zhan LT, Ling DS, Cleall PJ (2010) An efficient approach for locating the critical slip surface in slope stability analyses using a real-coded genetic algorithm. Can Geotech J 47(7):806-820 
22. Cheng YM, Li L, Lansivaara T, Chi SC, Sun YJ (2008) An improved harmony search minimization algorithm using different slip surface generation methods for slope stability analysis. Eng Optim 40(2):95-115

23. Kang F, Li J, Ma Z (2013) An artificial bee colony algorithm for locating the critical slip surface in slope stability analysis. Eng Optim 45(2):207-223

24. Khajehzadeh M, Taha MR, El-Shafie A, Eslami M (2012) A modified gravitational search algorithm for slope stability analysis. Eng Appl Artif Intell 25(8):1589-1597

25. Sakellariou MG, Ferentinou MD (2005) A study of slope stability prediction using neural networks. Geotech Geol Eng 23(4):419

26. Kaunda RB, Chase RB, Kehew AE, Kaugars K, Selegean JP (2010) Neural network modeling applications in active slope stability problems. Environ Earth Sci 60(7):1545-1558

27. Sengupta A, Upadhyay A (2009) Locating the critical failure surface in a slope stability analysis by genetic algorithm. Appl Soft Comput 9(1):387-392

28. Goh ATC (2003) Genetic algorithm search for critical slip surface in multiple-wedge stability analysis. Eng Optim 35:51-65

29. Zolfaghari AR, Heath AC, McCombie PF (2005) Simple genetic algorithm search for critical non-circular failure surface in slope stability analysis. Comput Geotech 32(3):139-152

30. Cheng YM, Li L, Chi SC (2007) Performance studies on six heuristic global optimization methods in the location of critical slip surface. Comput Geotech 34(6):462-484

31. Chen ZY, Shao CM (1988) Evaluation of minimum factor of safety in slope stability analysis. Can Geotech 」 25(4):735-748

32. Choobbasti AJ, Farrokhzad F, Barari A (2009) Prediction of slope stability using artificial neural network (case study: Noabad, Mazandaran, Iran). Arab J Geosci 2(4):311-319

33. Ni SH, Lu PC, Juang CH (1996) A fuzzy neural network approach to evaluation of slope failure potential. Comput Aided Civil Infrastruct Eng 11(1):59-66

34. SimSlope v.1.0—-slope stability analysis software. 2014

35. GeoSlope (2016) Slope stability analysis. SLOPE S/W

36. Alkasawneh W, Malkawi AlH, Nusairat JH, Albataineh N (2008) A comparative study of various commercially available programs in slope stability analysis. Comput Geotech 35(3):428-435

37. Rocscience (2002) Slide, a synopsis of slope stability analysis. Technical report, Rocscience

38. Yang XS, Deb S (2010) Cuckoo search via levy flights. In: Proc. of world congress on nature \& biologically inspired computing (NaBIC 2009), IEEE Publications, USA, pp 210-214

39. Kirkpatrick S (1984) Optimization by simulated annealing: quantitative studies. J Stat Phys 34:975-986

40. Malkawi AlH, Hassan WF, Sarma SK (2001) An efficient search method for finding the critical circular slip surface using the Monte Carlo technique. Can Geotech J 38(5):1081-1089

41. McCombie PF, Wilkinson P (2002) The use of the simple genetic algorithm in finding the critical factor of safety in slope stability analysis. Comput Geotech 29(8):699-714

42. Kashani AL, Gandomi AH, Mousavi M (2016) Imperialistic competitive algorithm: a metaheuristic algorithm for locating the critical slip surface in 2-dimensional soil slopes. Geosci Front 7(1):83-89

43. Mernik M, Liu SH, Karaboga D, Črepinšek M (2015) On clarifying misconceptions when comparing variants of the artificial bee colony algorithm by offering a new implementation. Inf Sci 291:115-127

44. Črepinšek M, Liu SH, Mernik L, Mernik M (2016) Is a comparison of results meaningful from the inexact replications of computational experiments? Soft Comput 20(1):223-235

45. Majumdar A, Maiti DK, Maity D (2012) Damage assessment of truss structures from changes in natural frequencies using ant colony optimization. Appl Math Comput 218(19):9759-9772

46. Mishra M, Maity D, Shakya A et al (2019) Structural health monitoring based on the hybrid ant colony algorithm by using Hooke-Jeeves pattern search. SN Appl Sci 1(7):799

47. Mishra M, Barman SK, Maity D, Maiti DK (2020) Performance studies of 10 metaheuristic techniques in determination of damages for large-scale spatial trusses from changes in vibration responses. J Comput Civil Eng 34(2):04019052

48. Eaton J, Yang S (2016) Railway platform reallocation after dynamic perturbations using ant colony optimisation. In: IEEE symposium series on computational intelligence (SSCI), pp 1-8

49. Ghazavi M, Bonab SB (2011) Optimization of reinforced concrete retaining walls using ant colony method. In: ISGSR - Vogt, Schuppener, Straub and Brau, ISBN 978-3-939230-01-4

50. Yu L, Xu P (2011) Structural health monitoring based on continuous aco method. Microelectron Reliability 51(2):270-278

51. Dias JC, Machado P, Silva DC, Abreu PH (2014) An inverted ant colony optimization approach to traffic. Eng Appl Artif Intell 36:122-133

52. Jabbarpour MR, Jalooli A, Shaghaghi E, Noor RM, Rothkrantz L, Khokhar RH, Anuar NB (2014) Ant-based vehicle congestion avoidance system using vehicular networks. Eng Appl Artif Intell 36:303-319

53. Chen XY, Chau KW, Busari AO (2015) A comparative study of population-based optimization algorithms for downstream river flow forecasting by a hybrid neural network model. Eng Appl Artif Intell 46:258-268

54. Cheng YM (2003) Location of critical failure surface and some further studies on slope stability analysis. Comput Geotech 30(3):255-267

55. Zhu DY, Lee CF, Qian QH, Zou ZS, Sun F (2001) A new procedure for computing the factor of safety using the morgenstern-price method. Can Geotech J 38(4):882-888

56. Dorigo M, Stützle T (2004) Ant colony optimization. Bradford Company, Scituate

57. Socha K, Dorigo M (2008) Ant colony optimization for continuous domains. Eur J Oper Res 185(3):1155-1173

58. Majumdar A, Nanda B, Maiti DK, Maity D (2014) Structural damage detection based on modal parameters using continuous ant colony optimization. Adv Civil Eng. https://doi.org/10.1155/2014/174185

\section{Publisher's Note}

Springer Nature remains neutral with regard to jurisdictional claims in published maps and institutional affiliations. 